\title{
False interpretation of diagnostic serology tests for patients treated with pooled human immunoglobulin G infusions: a trap for the unwary
}

\author{
Authors: Philip D Bright, ${ }^{\mathrm{A}}$ Lisa Smith, ${ }^{\mathrm{B}}$ Jane Usher, ${ }^{\mathrm{C}}$ Matthew Donati, ${ }^{\mathrm{D}}$ Sarah L Johnston, ${ }^{\mathrm{E}}$ Mark M Gompels ${ }^{\mathrm{F}}$ \\ and D Joe Unsworth ${ }^{\mathrm{G}}$
}

\begin{abstract}
Therapeutic immunoglobulin G (IgG) products are produced from numerous plasma donations, and are infused in many medical conditions. The serological testing of patients who have received IgG infusions may well produce falsely positive and misleading results from this infused IgG, rather than endogenously produced IgG. We present two example cases of clinical situations where this could cause concern. We tested multiple IgG products with a range of serological tests performed in infective or autoimmune conditions, including hepatitis B, syphilis, human immunodeficiency virus, human T-lymphotropic virus, anti-neutrophil cytoplasmic antibody (ANCA), anti-nuclear antibody (ANA), anti-cardiolipin antibodies and anti-double stranded DNA (dsDNA) antibody. We found positivity within these products for hepatitis B surface and core antibody, syphilis, ANCA, ANA, anticardiolipin IgG and dsDNA antibody, which may result from specific or non-specific reactivity. The serological testing of patients who have received IgG treatment detects the administered IgG in addition to IgG produced by the patient.
\end{abstract}

KEYWORDS: Serology, antibody, immunoglobulin, false positive

\section{Introduction}

Therapeutic immunoglobulin G (IgG) infusions are used in a wide variety of inflammatory, infective and immunodeficiency

Authors: Aconsultant clinical immunologist, Department of Immunology, Southmead Hospital, North Bristol NHS Trust, Bristol, UK; Bimmunology specialist nurse, Department of Immunology, Southmead Hospital, North Bristol NHS Trust, Bristol, UK; Cbiomedical head of virology Public Health England, Bristol Public Health Laboratory, Bristol, UK; ' D consultant virologist Public Health England, Bristol Public Health Laboratory, Bristol, UK, Econsultant clinical immunologist, Department of Immunology, Southmead Hospital, North Bristol NHS Trust, Bristol, UK; F consultant clinical immunologist, Department of Immunology, Southmead Hospital, North Bristol NHS Trust, Bristol, UK; ${ }^{G}$ Consultant clinical immunologist, Department of Immunology, Southmead Hospital, North Bristol NHS Trust, Bristol, UK disorders, and contain only trace amounts of $\operatorname{IgA}$ and IgM. Large doses (eg $2 \mathrm{~g} / \mathrm{kg}$ ) are used in the context of immunomodulatory therapy and the half-life of IgG is several weeks. IgG products are prepared from a large number of human plasma donations (eg 100-60,000 donations). Many routine laboratory tests for infective or autoimmune conditions are reliant on serological methods of testing for binding of specific IgG to a particular antigen. Serology results obtained from patients treated with pooled IgG will reflect both infused IgG antibodies contained in the administered IgG as well as endogenously produced IgG from the patient. IgG antibody tests on such patients within six weeks of IgG administration are generally unhelpful and sometimes dangerously misleading. We present two example cases below.

\section{Example cases}

\section{Case 1}

A 34 year-old female patient was referred to gastroenterology by rheumatology with an unexplained raised alanine aminotransferase (ALT) of $200 \mathrm{U} / \mathrm{l}$ (normal range 5-40 U/1). This patient had antibody deficiency secondary to gold therapy and multiple other disease modifying immunosuppressive agents for rheumatoid arthritis. Increased bacterial infections justified anti-infective prophylaxis including intravenous IgG (Privigen 20 g every 3 weeks). Her IgG on this treatment was usually within the normal range, but her IgM and IgA were very low, indicating that she was unlikely to be producing much endogenous antibody. Investigations for the cause of this hepatitis included serology for infectious hepatitis (Roche Cobas e 411 method) and revealed:

> IgG hepatitis B surface antibody strongly positive $>100 \mathrm{mIU} / \mathrm{ml}$

> IgG/IgM hepatitis B core antibody positive

$>$ hepatitis B surface antigen negative.

The virology laboratory report read: 'Antibody to hepatitis B due to naturally acquired past infection. At risk of reactivation if immunocompromised. Consider discussion with viral hepatitis service.'

In this immunocompromised context this raised concern, including the possibility of reactivation of hepatitis B disease in the future. Hepatitis B virus (HBV) nucleic acid testing by polymerase chain reaction was negative. A liver biopsy 
showed non-diagnostic inflammatory changes in the portal tracts and parenchyma, associated with mild steatosis and a modest periportal fibrosis. The histopathologist could not exclude hepatitis B as a cause of these changes. Subsequent multidisciplinary discussions involving the immunology team led to the conclusion that active hepatitis B was very unlikely. Pooled normal human IgG will contain hepatitis B surface antibody. The positive core antibody test may well be the result of previously cleared hepatitis B infection in donors.

\section{Case 2}

A 32-year-old female patient on treatment for antibody deficiency with replacement intravenous IgG (Vigam 30 g every 2 weeks) became pregnant. As part of the routine screening for her pregnancy she was tested for syphilis, yielding the following profile:

> syphilis rapid plasma reagin (RPR) negative

> syphilis treponema pallidum particle aggulutination (TPPA) negative

> syphilis IgM enzyme immunoassay (EIA) negative

$>$ syphilis total antibody EIA positive

> syphilis IgG immunoblot indeterminate.

Syphilis serology was repeated on a further sample and confirmed the original findings. The obstetric team were understandably concerned by the results, which were relayed to the patient. On discussion between the treating immunologist and virologist, the results were thought to represent false positives secondary to the IgG replacement therapy. Treatment was considered and discussed at length with the patient, and it was agreed that further antibiotics were not necessary over and above those previously received to treat confirmed infections associated with the antibody deficiency.

We suspected that the positive serological results in the example cases were the result of reactivity from administered IgG. Plasma donors for pooled human IgG are tested for antibodies to HIV, syphilis and hepatitis C virus (HCV); HBV surface antigen; and the viral nucleic acids of HIV, HBV, $\mathrm{HCV}$, hepatitis A and parvovirus B19. Individual plasma units, minipools and the final plasma product are also variously tested for viral antibodies, HBV surface antigen and viral nucleic acids. The companies that produce these IgG products do not test syphilis serology or hepatitis B core antibodies in the finished IgG product. Donors who have previously been exposed to hepatitis B and cleared it, and therefore have positive hepatitis $B$ surface and core antibodies with negative hepatitis B surface antigen, are therefore eligible to donate. We therefore tested a number of commercial IgG products with a variety of different serological laboratory assays, including some HBV markers.

\section{Results}

To ensure that the positive results for hepatitis B core antibody were not a result of one particular routine serological laboratory method or one particular IgG commercial product we tested seven undiluted IgG products using kits from different companies (Table 1).

We then tested five IgG products for antibodies to hepatitis B, syphilis, HIV and human T-lymphotropic virus (HTLV). To cover a limited number of common autoimmune serological
Table 1. Testing of undiluted IgG products for HBV core $\mathrm{Ab}$ by different company kits.

\begin{tabular}{llll} 
& $\begin{array}{l}\text { Roche } \\
\text { (Cobas } \\
\text { system) }\end{array}$ & $\begin{array}{l}\text { Ortho-clinical } \\
\text { diagnostics } \\
\text { (Vitros } \\
\text { system) }\end{array}$ & $\begin{array}{l}\text { Biomerieux } \\
\text { (VIDAS } \\
\text { system) }\end{array}$ \\
$\begin{array}{l}\text { Octagam } \\
\text { Privigen }\end{array}$ & Pos & Pos & Pos \\
Flebogamma DIF & Pos & Pos & Not done \\
Kiovig & Pos & Pos & Pos \\
Vigam & Pos & Not done & Pos \\
Gammaplex & Pos & Not done & Pos \\
Subcuvia & Pos & Not done & Pos \\
\hline Ab=antibody; HBV = hepatitis B virus; IgG =immunoglobulin G; Pos = positive.
\end{tabular}

investigations we tested the products for anti-neutrophil cytoplasmic antibodies (ANCA), anti-nuclear antibodies (ANA), anti-cardiolipin IgG and IgM (positive in the antiphospholipid syndrome) antibodies and anti-double stranded DNA (dsDNA) antibodies (Table 2). Results are given for the neat product and for diluted product to a physiological concentration of $8 \mathrm{~g} / \mathrm{l}$ by dilution in distilled water. $8 \mathrm{~g} / \mathrm{l}$ was chosen as it represents a reasonable target trough concentration of IgG in blood in patients on replacement immunoglobulin products.

\section{Discussion on hepatitis B core antibody testing}

Positive HBV core antibody was found for all IgG products tested at neat concentration by all three different methods tested. At physiological concentrations, all IgG products tested were positive for HBV core antibody apart from Kiovig (where the two results were discrepant - see Table 2). HBV surface antibody was strongly positive for all IgG products tested at both neat and physiological concentrations, and HBV surface antigen was uniformly negative. There are published examples of similar cases to case 1 , such as a patient on IgG with a positive HBV core antibody who was negative on repeat testing 4 weeks later once the IgG was stopped, ${ }^{1}$ and a spuriously positive $\mathrm{HBV}$ core antibody and $\mathrm{HBV}$ surface antigen for a patient on IgG treatment. ${ }^{2}$

Occult HBV infection detected by HBV DNA testing has been shown in $0.1-2.4 \%$ (median $1 \%$ ) of HBV surface antigennegative, HBV core antibody-positive blood donors in Western countries such as the United States. ${ }^{3}$ HBV transmission by blood components negative for HBV surface antigen has occurred historically, ${ }^{4-6}$ but is highly unlikely with current manufacturing methods. Risk of transmission of hepatitis B from plasma products appears to be negligible when concurrent HBV surface antibody is present in the blood above a certain level (100-200 mIU/ml), and it has been observed that blood containing detectable HBV surface antibody carries no increased risk of transmitting hepatitis B when compared with blood that lacks this antibody. ${ }^{7}$ Screening and prevention of blood donations from those donors positive for HBV core antibody is only feasible in areas of low endemic disease. Since commercial companies do not screen donors for HBV core antibody but do screen for HBV DNA and HBV surface antigen, 


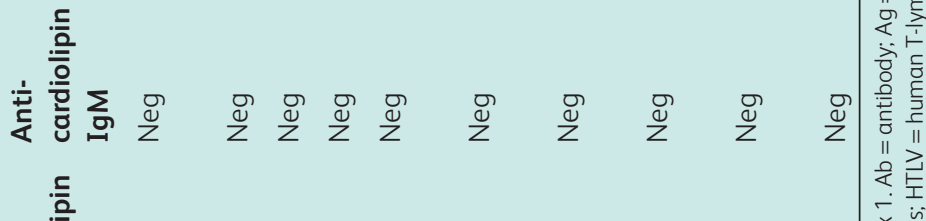

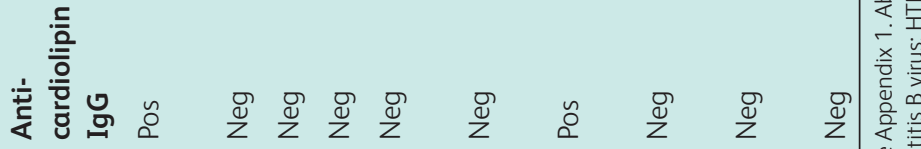

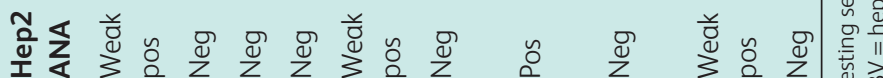

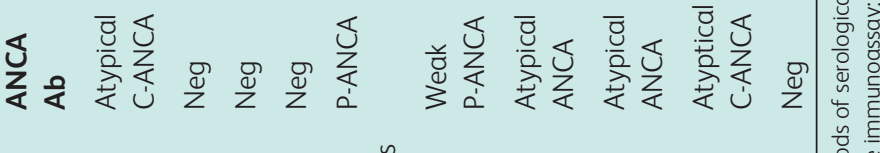

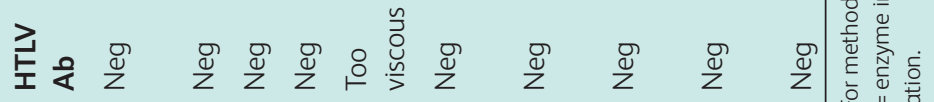

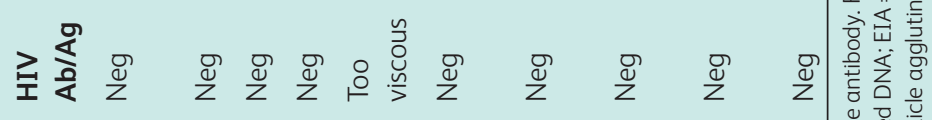

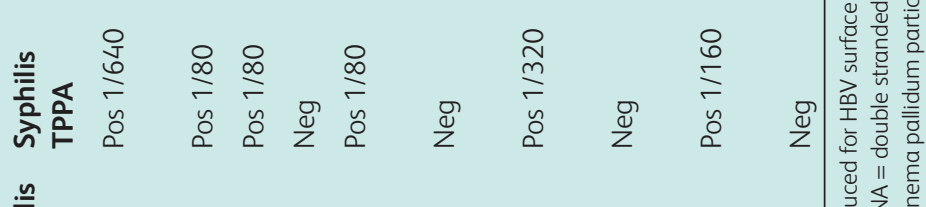

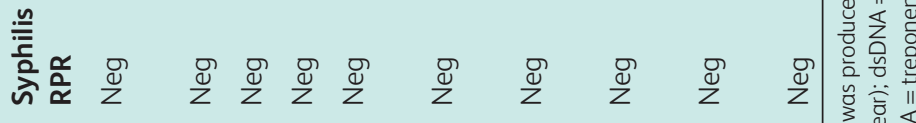

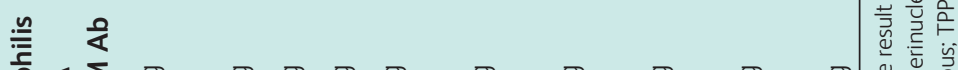

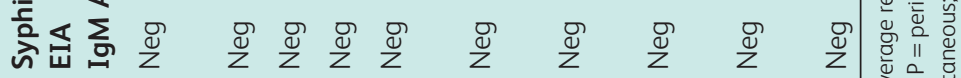

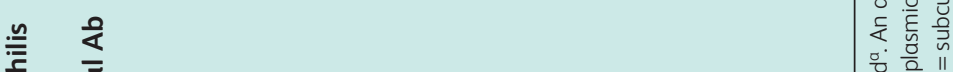

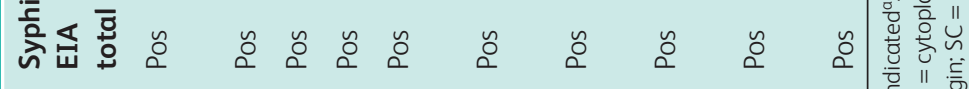

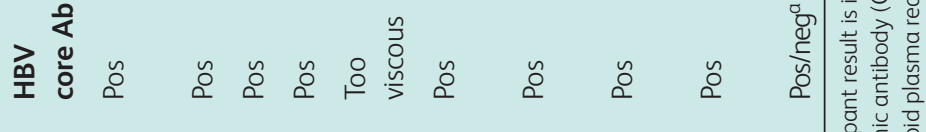

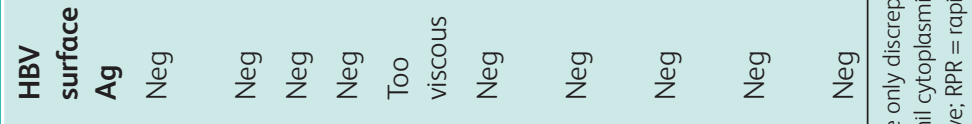

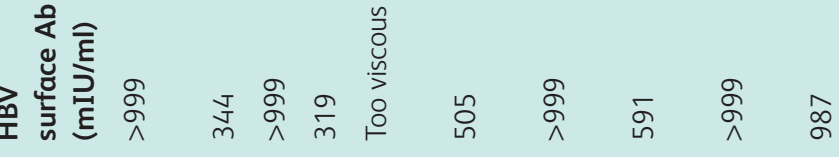


it is possible that the positivity in commercial IgG reflects specific antibody from donors with cleared HBV infection.

A particular clinical scenario that could significantly impact patient care would be in a patient being prepared for immunosuppression, such as in the context of idiopathic thrombocytopaenic purpura (ITP). These patients are frequently treated with IgG and/or rituximab. Rituximab may cause reactivation of $\mathrm{HBV}$ even in patients with remote historical infection, ${ }^{8-12}$ so testing for HBV serology prior to rituximab treatment is advised. In the context of IgG treatment, falsely positive HBV core antibody serology is likely and would complicate the decision to use rituximab therapy. Arnold et al ${ }^{13}$ published a case series on this clinical situation which indicated 11 ITP cases were seropositive for hepatitis B core antibody, of which 10 had received IgG. Seven of these 10 patients subsequently reverted to negative hepatitis B core antibody on repeat testing having discontinued IgG.

\section{Discussion on syphilis serological testing}

The syphilis serological results of commercial IgG products showed uniform negativity for syphilis enzyme immunoassay $\operatorname{IgM}$, which is unsurprising given there is a negligible amount of IgM in IgG products (which are over 99\% IgG). However, syphilis testing of products was uniformly positive at neat and physiological concentrations for the syphilis enzyme immunoassay for total (IgG and IgM) reactivity. The IgG TPPA results showed some disparity, with most neat solutions producing a positive result and most physiological products producing a negative result. It has previously been reported that there have been false positive serological results for syphilis in patients on IgG treatment. Constable et a ${ }^{14}$ reported a patient on IgG treatment for Guillain-Barre syndrome with positive syphilis serology (EIA and TPPA, but negative RPR) 24 hours after infusion, with subsequent testing at 8 and 13 weeks following infusion being negative. Similarly, Rossi et al ${ }^{15}$ described a pregnant patient who tested positive for syphilis from IgG therapy for neonatal alloimmune thrombocytopenia. This patient had negative serology testing prior to and 6 weeks following her last treatment, but she received antibiotic treatment during pregnancy for syphilis anyway.

In view of the screening questions, medical examination and serological screening for syphilis in donor populations, it is likely that the above positive syphilis serological results are the result of non-specific reactivity, as opposed to the possible true positivity for HBV core antibody. It is unlikely that there is a significant burden of syphilis in the donor populations as donors are usually regularly tested for syphilis. There remains a question as to whether treatment of syphilis in these patients during pregnancy is warranted in view of the likelihood of it relating to the infused IgG. The treatment for syphilis is safe and effective, and the outcomes of active syphilis for the fetus are potentially severe (stillbirth or severe disability). This decision should be made on an individual basis for each patient after taking a thorough sexual history.

\section{Discussion on other microbiological serological tests}

Serological testing of products for HIV and HTLV antibody were uniformly negative. False positive tests for toxoplasma serology have been found in patients on IgG treatment previously, ${ }^{16}$ and would be expected from a normal population of donors not screened for this infection.

\section{Discussion on rheumatological serological testing}

At physiological concentrations, most of the autoantibody tests were negative. Weak positive ANCA results were seen for two of the products. At neat (non-physiological) concentrations, ANCA, ANA, anti-cardiolipin IgG and anti-dsDNA variably tested positive. This is suggestive of non-specific binding in these assays, but could reflect low level positivity of these antibodies within the IgG products. Since there are only trace amounts of IgM in IgG products, the uniform negativity of testing products for IgM anti-cardiolipin antibody is expected.

\section{Conclusion}

The serological testing of patients on IgG treatment tests for the presence of specific antibody in the IgG product, in addition to the endogenous specific antibody of the patient. False positivity on serological testing may also result from non-specific reactivity from donor IgG. Results should therefore be interpreted with care, especially when positive, as they may well be falsely so. Knowledge of the manufacturing and testing processes and the likely seropositivity of large donor pools of IgG allow for some interpretation of results, but we suggest that in some situations, serological testing should simply be avoided in patients on IgG (eg HBV core antibody), especially those receiving high dose therapy. Delayed repeat serological testing weeks after IgG administration may show low or absent positivity on a given serological test, consistent with a passively acquired effect.

\section{Summary points}

> IgG is detectable for some weeks after administration, and serology tests during this time will identify the administered IgG in addition to endogenously produced IgG.

> This may result in false positivity to a range of serological tests utilised in infective or autoimmune conditions, such as hepatitis B serology, syphilis serology and ANCA.

\section{Competing interests}

PDB received travel sponsorship for a conference from CSL Behring. LS received conference and travel sponsorship from CSL Behring, Grifols and Baxter. MD received travel sponsorship from Gilead and JanssenCilag, educational meeting support from Pfizer and Hologic, and lecture fees from Sanofi-Pasteur MSD. MG attended advisory boards, lectured for, and received travel scholarships for CSL Behring and Shire group.

\section{References}

1 Benton E, Iqbal K, Wade P et al. False-positive hepatitis B serology following IVIG therapy: forgotten but not gone!! J Am Acad Dermatol 2012;66:e123-4.

2 Lichtiger B, Rogge K. Spurious serologic test results in patients receiving infusions of intravenous immune gammaglobulin. Arch Pathol Lab Med 1991;115:467-9.

3 Hollinger FB. Hepatitis B virus infection and transfusion medicine: science and the occult. Transfusion 2008;48:1001-26.

4 Satake M, Taira R, Yugi H et al. Infectivity of blood components with low hepatitis B virus DNA levels identified in a look back program. Transfusion 2007;47:1197-205. 
5 Gerlich WH, Wagner FF, Chudy M et al. HBsAg non-reactive HBV infection in blood donors: transmission and pathogenicity. J Med Virol 2007;79:S32-6.

6 Hoofnagle JH, Seeff LB, Bales ZB, Barker LF. Type B hepatitis after transfusion with blood containing antibody to hepatitis B core antigen. N Engl J Med 1978;298:1379-83.

7 Aach RD, Alter HJ, Hollinger FB et al. Risk of transfusing blood containing antibody to hepatitis B surface antigen. Lancet 1974;2:190-3.

8 Dai MS, Chao TY, Kao WY, Shyu RY, Liu TM. Delayed hepatitis B virus reactivation after cessation of preemptive lamivudine in lymphoma patients treated with rituximab plus CHOP. Ann Hematol 2004;83:769-74.

9 Dervite I, Hober D, Morel P. Acute hepatitis B in a patient with antibodies to hepatitis B surface antigen who was receiving rituximab. N Engl J Med 2001;344:68-9.

10 Westhoff TH, Jochimsen F, Schmittel A et al. Fatal hepatitis B virus reactivation by an escape mutant following rituximab therapy. Blood 2003;102:1930.

11 Yeo W, Chan TC, Leung NW et al. Hepatitis B virus reactivation in lymphoma patients with prior resolved hepatitis B undergoing anticancer therapy with or without rituximab. J Clin Oncol 2009;27:605-11.
12 Lok AS, McMahon BJ. Chronic hepatitis B. Hepatology 2007;45:507-39.

13 Arnold DM1, Crowther MA, Meyer RM et al. Misleading hepatitis $\mathrm{B}$ test results due to intravenous immunoglobulin administration: implications for a clinical trial of rituximab in immune thrombocytopenia. Transfusion 2010;50:2577-81.

14 Constable SA, Parry CM, Enevoldson TP, Bradley M. Positive serological tests for syphilis and administration of intravenous immunoglobulin. Sex Transm Infect 2007;83:57-8.

15 Rossi KQ, Nickel JR, Wissel ME. Passively acquired treponemal antibody from intravenous immunoglobulin therapy in a pregnant patient. Arch Pathol Lab Med 2002;126:1237-8.

16 Pelloux H, Fricker-Hidalgo H, Goullier-Fleuret A, AmbroiseThomas P. Detection of anti-Toxoplasma immunoglobulin $\mathrm{M}$ in pregnant women. J Clin Microbiol 1997;35:2187.

Address for correspondence: Dr PD Bright, Department of Immunology, Pathology Sciences, Southmead Hospital, North Bristol NHS Trust, Westbury on Trym, Bristol BS10 5NB, UK. Email: philip.bright@doctors.org.uk

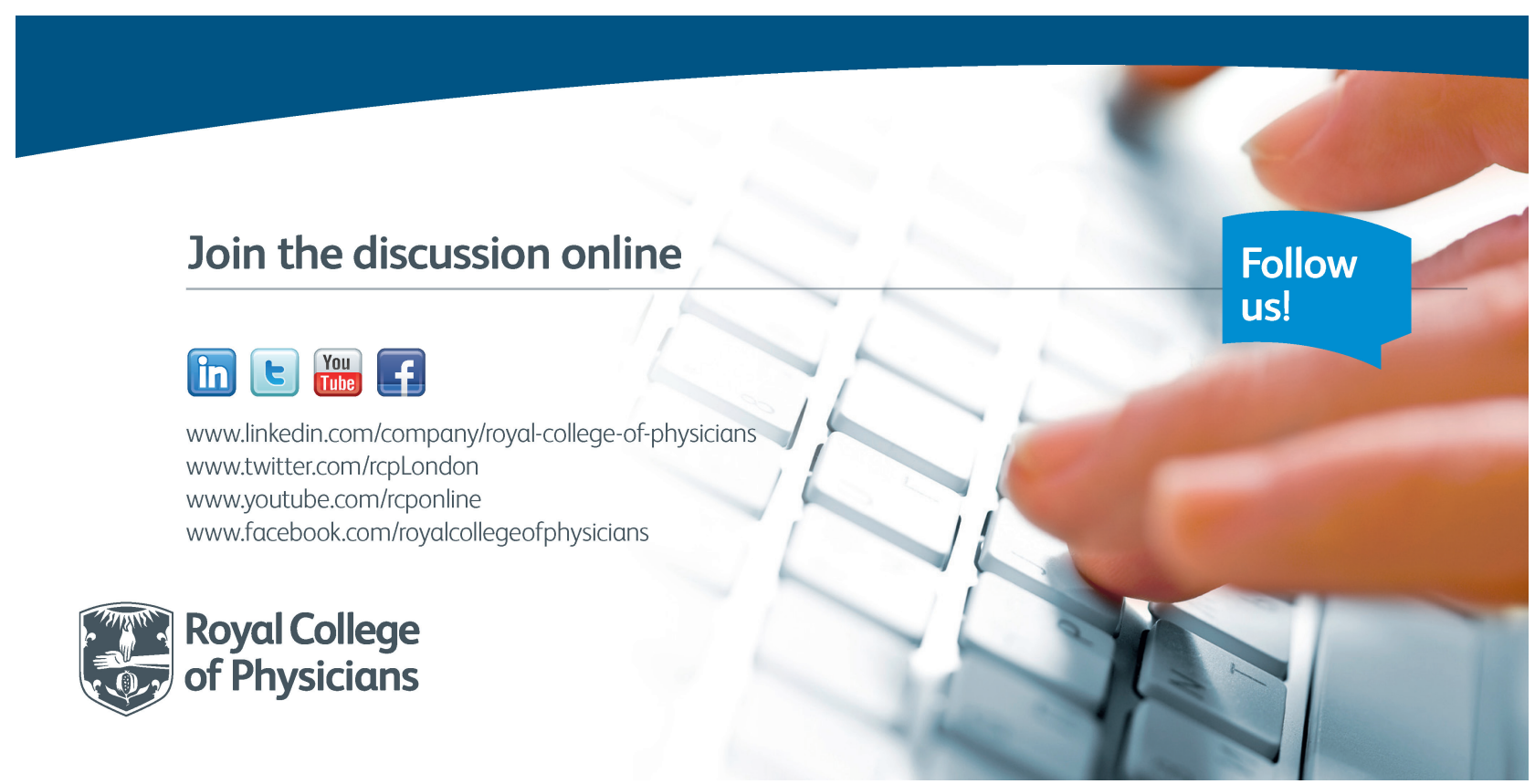

